\title{
A Definite Explanation of the Concept of Limit in Teaching
}

\author{
Wenbing $\mathrm{Wu}$ \\ Nanchang Normal University, Nanchang, China \\ Email: wwbysq@fjnu.edu.cn
}

How to cite this paper: $\mathrm{Wu}, \mathrm{W}$. B. (2020). A Definite Explanation of the Concept of Limit in Teaching. Creative Education, 11, 2832-2836

https://doi.org/10.4236/ce.2020.1112208

Received: November 7, 2020

Accepted: December 21, 2020

Published: December 24, 2020

Copyright $\odot 2020$ by author(s) and Scientific Research Publishing Inc. This work is licensed under the Creative Commons Attribution International License (CC BY 4.0).

http://creativecommons.org/licenses/by/4.0/

\begin{abstract}
The limit idea is the basic idea of calculus. Almost all concepts in mathematical analysis are inseparable from the limit, but the limit is a concept that is difficult to understand accurately. By assuming that there is a certain point closest to the limit value, this paper provides a reasonable explanation for the infinitesimal paradox and a new answer to the question why the limit value is accurate in teaching. At the same time, this method is applied to the derivative and used to understand a common practical problem in mathematics. The analysis shows that this method is effective for the accurate understanding of limit problems.
\end{abstract}

\section{Keywords}

Limit, Infinitesimal Paradox, Interpretation, Determination

\section{Introduction}

The limit idea is the basic idea of calculus. A series of important concepts in mathematical analysis, such as the continuity of the function, the derivative (to get the maximum value for 0 ), and the definite integral are all defined with the help of limits. If you want to ask: "What kind of subject is mathematical analysis?" Then you can say in a nutshell: Mathematical analysis is a subject that uses limit thinking to study functions, and the error of the calculation result is so small that it is hard to imagine, so it can be ignored.

Liu Hui's circle-cutting technique is an original and reliable application of the limit idea of "keep approaching" based on the study of intuitive graphics; in the 16th century, the Dutch mathematician Stevin made improvements in the process of investigating the center of gravity of triangles. The ancient Greek's method of exhaustion, he used geometric intuition to boldly use limit thinking to think about problems, and gave up the proof of Guimufa. In this way, he inadvertently 
"pointed out the direction of developing the limit method into a practical concept."

The further development of limit thinking is closely related to the establishment of calculus. Newton and Leibniz established calculus based on the concept of infinitesimals. Later, due to logical difficulties, they accepted the limit idea to varying degrees in their later stages. Newton used the ratio $\frac{\Delta s}{\Delta t}$ of the change in distance $\Delta S$ to the change in time $\Delta t$ to express the average speed of a moving object, let $\Delta t$ approach zero infinitely, and obtain the instantaneous speed of the object, and derived the concept of derivative and differential theory from this. He realized the importance of the concept of limit and tried to use the concept of limit as the basis of calculus, but Newton's concept of limit was also based on geometric intuition, so he could not draw a strict expression of the limit.

Limit thinking has a wide range of applications in modern mathematics and even physics and other disciplines, which is determined by its inherent thinking function. Limit thinking reveals the unity of opposites between variables and constants, and between infinity and finite. It is the application of the law of unity of opposites of materialist dialectics in the field of mathematics. With the help of limit thinking, people can understand the infinite from the finite, the "change" from the "unchanged", the "curved shape" from the "straight line", the qualitative change from the quantitative change, and the precise understanding from the approximation.

People can scientifically determine the extremely accurate value of that quantity by investigating the trend and trend of a series of increasingly precise approximations of certain functions. This requires the use of the concept of limit and the above limit thinking method. The limit thinking method is scientific, because extremely accurate conclusions can be obtained through the limit function calculation method.

At present, there are also many documents actively discussing the meaning of limits and the teaching methods of limits (Xiao, 1982; Chen \& Mi, 2001; Nie \& Chang, 2010; Zhang, 2019), and the literature (Wang \& Jin, 2018; Zhang, 2019) has conducted a strict theoretical discussion on limits. On the basis of these discussions, this article attempts to find a method that can not only make the concept and thought of the limit easier to understand, but also can explain the abovementioned "infinitesimal paradox" and why the limit is accurate. Wait for more difficult concepts.

\section{New Ways to Understand the Limit}

It is more difficult to accurately grasp the concept of "infinite proximity but never equal" in the limit, because infinite proximity is an uncertain concept. This article attempts to use a certain method to understand the meaning of infinite proximity. For the simplest example, for example,

$$
\lim _{x \rightarrow 0} x=0
$$


First of all, this result is undoubtedly correct. Second, in this article, the $x$ in the formula (1) is regarded as a pointer, and the position it points to is the value of the variable $x$. As the pointer $x$ approaches 0 , the variable $x$ will infinitely approach 0 , but it will never be equal to 0 . When the pointer $x$ approaches 0 , the variable $x$ can be equal to $0.1,0.01,0.001$, etc. How do we understand this infinite process? No matter 0.1 , or $0.01,0.001 \ldots$ all correspond to a point on the number axis respectively. This infinite approaching process is actually the process in which the pointer $x$ on the number axis $\mathrm{X}$ gradually approaches 0 . So, can it be considered that there is a point on the $\mathrm{X}$ axis that is closest to 0 ? According to geometric axioms, a straight line is composed of points one by one. Based on this, it can be determined that the point closest to 0 exists. Although this point cannot determine its position, that is, it is impossible to determine what the number representing the coordinate of this point is, because if Say that point a (you can use a number to indicate its position) is closest to 0 , then there must be other points between 0 and a (there must be other rational or irrational numbers between two rational numbers or two irrational numbers). In order to understand the concept of limit, this article does not care whether this point a can be represented by a certain number, as long as it is considered to exist. With the premise of this hypothesis, then it becomes easy to understand the concept of limit (Figure 1 \& Figure 2).

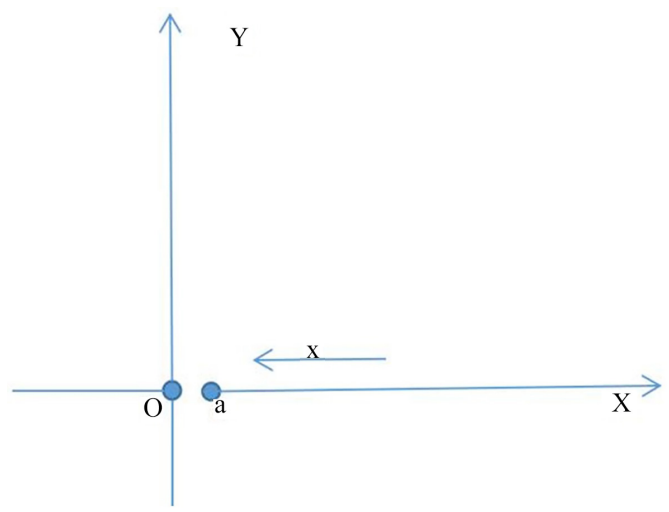

Figure 1. Limit explanation 1.

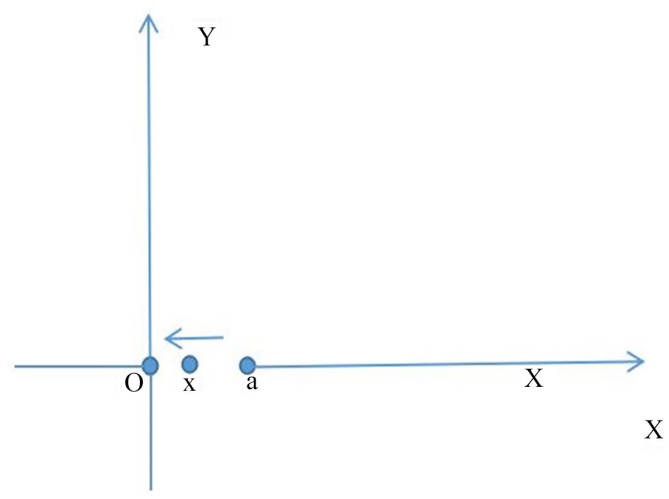

Figure 2. Limit explanation 2. 
First of all, this result is undoubtedly correct. Second, in this article, the $x$ in the formula (1) is regarded as a pointer, and the position it points to is the value of the variable $x$. As the pointer $x$ approaches 0 , the variable $x$ will infinitely approach 0 , but it will never be equal to 0 . When the pointer $x$ approaches 0 , the variable $x$ can be equal to $0.1,0.01,0.001$, etc. How do we understand this infinite process? No matter 0.1 , or $0.01,0.001 \ldots$ all correspond to a point on the number axis respectively. This infinite approaching process is actually the process in which the pointer $x$ on the number axis $\mathrm{X}$ gradually approaches 0 . So, can it be considered that there is a point on the $\mathrm{X}$ axis that is closest to 0 ? According to geometric axioms, a straight line is composed of points one by one. Based on this, it can be determined that the point closest to 0 exists. Although this point cannot determine its position, that is, it is impossible to determine what the number representing the coordinate of this point is, because if Say that point a (you can use a number to indicate its position) is closest to 0 , then there must be other points between 0 and a (there must be other rational or irrational numbers between two rational numbers or two irrational numbers). In order to understand the concept of limit, this article does not care whether this point a can be represented by a certain number, as long as it is considered to exist. With the premise of this hypothesis, then it becomes easy to understand the concept of limit.

This hypothesis can also more accurately explain why Cauchy considers infinitesimals as "variables with 0 as the limit", and explains why the concept of "infinitesimals" is not like zero but can be artificially treated as equal to 0 . Figure 2 shows that in the process of variable change, its value is actually not equal to zero, but its changing trend is "zero", which can be infinitely close to zero. Therefore, it can be processed with "equal to 0 " without producing wrong results.

If formula (1) is understood in this way, the limit becomes a definite concept. Because, according to this understanding, the limit can be expressed as: this limit equation is when the pointer $x$ is between 0 and the point a closest to it, and when the pointer $x$ is at the end close to point 0 , that is, pointer $x$ and point 0 When the distance between the two is less than half of the gap between point 0 and point a, this equation holds.

The main point of this method is that it is assumed that the point closest to the limit value is certain, thereby turning the limit idea with uncertain meaning into a certain concept.

\section{Conclusion}

The concept of limit is a basic mathematical concept, and its thinking has a certain degree of uncertainty. Therefore, it is very difficult to understand this concept. This article follows the axiom that a straight line is composed of points, assuming that the limit is away from the limit. The point with the nearest value is certain to exist, regardless of whether it can be represented by a number. Through this assumption, the uncertainty in the limit thought can be transformed into a 
definite concept, which is a teaching sense for the understanding of the limit. A new method has been added, which also reduces the difficulty of understanding the concept of limit. This method of understanding can also successfully explain other mathematical concepts and phenomena.

\section{Acknowledgements}

This paper is supported by Research Foundation of the Nanchang Normal University for Doctors (NSBSJJ2018014). Key R\&D Project of Jiangxi Provincial Department of Science and Technology (20192BBEL50040, 20192BBHL80002).

\section{Conflicts of Interest}

The author declares no conflicts of interest regarding the publication of this paper.

\section{References}

Chen, G., \& Mi, P. Z. (2001). Research on the Limit Thinking in Advanced Mathematics. College Mathematics, 17, 69-71.

Nie, L. C., \& Chang, N. L. (2010). Exploration and Analysis of Limit Concept Teaching in Advanced Mathematics. Journal of Baoding University, 23, 131-134.

Wang, J. Z., \& Jin, Y. R. (2018). Discussion on the Limit of a Sequence of Numbers. College Mathematics, 4, 115-117.

Xiao, B. R. (1982). A Preliminary Discussion on Limit, Derivative and Differential Teaching in Middle School Mathematics. Journal of Nanjing Normal University (Natural Science Edition), 3, 76-78.

Zhang, Q. L. (2019). Analysis and Breakthrough of the Difficulties in Advanced Mathematics Limit Concept Teaching. Mathematics Learning and Research: Teaching Research Edition, 3, 32.

Zhang, W. B. (2019). Some Thoughts on the Introduction of Mathematics Culture into the Teaching of Limit Concept. Mathematics Learning and Research, 9, 30. 